\title{
Miosite ossificante progressiva: uma perspectiva no controle da doença
}

\author{
A perspective on the control of myositis ossificans progressiva
}

\author{
Durval B. Palhares ${ }^{1}$, Lígia M. Leme ${ }^{2}$
}

\section{Resumo}

Objetivo: relatar quatro casos de miosite ossificante progressiva, forma de apresentação da doença e novas medidas de controle e tratamento.

Descrição: os autores descrevem quatro casos de miosite ossificante progressiva. Apresentam as manifestações clínicas e evolução da doença e as formas de controle e tratamento disponíveis. Nos quatro casos são descritas as principais anomalias congênitas associadas: halux valgo, clinodactilia, polegares curtos, osteófitos e sindactilia. Descrevem-se a evolução e a resposta terapêutica de melhora clínica ao uso oral de ácido ascórbico e bifosfonado nos pacientes 1 e 4; entretanto, o paciente 1 obteve uma melhor resposta após receber bifosfonado endovenoso. Os pacientes 2 e 3 melhoraram clinicamente tomando apenas ácido ascórbico oral.

Comentários: o ácido ascórbico diminui a dor e melhora o psiquismo do paciente com miosite ossificante progressiva. Bifosfonado endovenoso é uma alternativa no paciente com piora importante, principalmente com limitação de deambulação. A orientação para se evitar trauma repetitivo nos pacientes com miosite ossificante progressiva é importante.

J Pediatr (Rio J) 2001; 77 (5): 431-4: miosite ossificante progressiva, ácido ascórbico, ossificação ectópica, bifosfonado.

\begin{abstract}
Objective: to report four cases of myositis ossificans progressiva, its form of presentation, in addition to new control and treatment options.

Description: the authors describe four cases of myositis ossificans progressiva, presenting its clinical manifestations, evolution, and available forms of control and treatment. The main congenital anomalies associated with myositis ossificans progressiva are described, namely: hallux valgus, clinodactyly, short thumbs, exostosis, and syndactyly. The evolution and therapeutic responses of clinical improvement to oral ascorbic acid and biphosphonate in patients 1 and 4 are also described; patient 1 had a better response after receiving intravenous biphosphonate, while patients 2 and 3 showed clinical improvement after the administration of oral ascorbic acid.

Comments: Ascorbic acid relieves the pain and improves psychism in patients with myositis ossificans progressiva. Intravenous biphosphonate is an alternative to patients with significant worsening of the disease, especially those with ambulatory restrictions. The orientation towards avoiding recurrent trauma in myositis ossificans progressiva patients is important.
\end{abstract}

J Pediatr (Rio J) 2001; 77 (5): 431-4: myositis ossificans progressiva, ascorbic acid, ectopic ossifications, biphosphonate.

\section{Introdução}

A Miosite Ossificante Progressiva (MOP) ou Fibrodisplasia ossificante progressiva é uma doença rara, com menos de 1.000 casos descritos, autossômica dominante e com expressividade variável, mas muitos casos são esporá$\operatorname{dicos}^{1}$. Foi descrita pela primeira vez por Patin ${ }^{2}$. A maioria dos pacientes apresenta, como peculiaridades características, altas taxas de anomalias congênitas associadas mais freqüentemente ao polegar e halux, presentes ao nascimento em $75 \%$ a $90 \%$ dos casos. Outras alterações incluem: adactilia, microdactilia, ancilose interfalangeana e clino-

1. Prof. Adjunto Doutor - Departamento de Pediatria.

2. Profa Adjunta Doutora do Departamento de Clínica Médica.

Universidade Federal de Mato Grosso do Sul - Campo Grande, MS.

dactilia, canal lombar estreito, ângulo úmero-epicondilar diminuído e pseudoexostoses. A formação de ossos ectópicos envolve tendões, fáscia e aponeuroses, ocorrendo em torno dos músculos esqueléticos estriados.

A fisiopatologia da doença é pouco conhecida. A manifestação inicial caracteriza-se por edema de partes moles, sendo a região da nuca a mais freqüente ${ }^{3}$, porém outras regiões, como as pernas e o dorso podem fazer parte do quadro inicial. A doença caracteriza-se por freqüentes edemas, conseqüentes a processos inflamatórios, que vão surgindo e posteriormente vão se calcificando, e o paciente aos poucos vai perdendo a mobilidade da região afetada. Trauma tem sido um dos fatores de agudização da doença, embora alguns episódios têm sido observados sem traumatismo como fator desencadeante. 
Inúmeros tratamentos têm sido preconizados: administração oral de etidronato dissódico (EHDP) com variáveis respostas ${ }^{4-6}$; uso de esteróides, isotretinoína, etc. Estes tratamentos têm demonstrado pouca eficácia no controle da doença. Os bifosfonados, como o EHDP, clodronato, são inibidores do metabolismo ósseo e resistentes à hidrólise enzimática. Sua ação farmacológica principal é a redução da reabsorção óssea normal e anormal. O mecanismo de ação dos bifosfonados na prevenção da ossificação heterotópica não é conhecido. Estudos químicos e in vitro mostram que esta droga adsorve os cristais de hidroxiapatita, diminuindo a formação heterotópica óssea durante o estágio ativo da doença ${ }^{7}$.

Palhares $(1997)^{8}$ observou que o ácido ascórbico, em altas doses, controlou a progressão da doença. Nesse artigo há relatos da evolução de outros pacientes com o tratamento com ácido ascórbico.

As medicações prescritas já são utilizadas na MOP e em outras doenças. A associação do ácido ascórbico com os bifosfonados foi utilizada com sucesso em um paciente (caso 1), levando-se em consideração os benefícios isolados de cada um destes medicamentos. Houve consentimento dos pais. Através de comentários com colegas médicos, outros pais de pacientes portadores de MOP nos procuraram solicitando este tratamento.

O objetivo do trabalho é relatar quatro casos de pacientes com MOP e propor novas alternativas no controle e tratamento desta doença com o ácido ascórbico e bifosfonado.

\section{Relato de Casos \\ Caso 1}

A.G.P., 16 anos de idade, masculino, nascido a termo, gestação sem intercorrências. Assintomático até 30 meses de idade. Portador de halux valgo bilateral e clinodactilia. Início dos sintomas com o aparecimento de torcicolo de difícil controle acompanhado de dor intensa e edema, contraturas e ossificações musculares nos membros superiores e região torácica. Seis anos e meio após o início dos sintomas, foi iniciado o tratamento com ácido ascórbico ${ }^{8}$, observando-se desaparecimento do quadro doloroso, regressão dos edemas e estabilização das ossificações (Figura 1). Permaneceu assintomático até julho/2000, sob tratamento contínuo com ácido ascórbico $500 \mathrm{mg}, 4$ vezes ao dia e um bifosfonado (alendronato de sódio), que foi substituído pelo etidronato dissódico (didronelâ), 400mg/dia, conseqüente a epigastralgia. Após essa época o paciente apresentou crise de MOP com edema, dor e ossificação ao nível da musculatura do fêmur esquerdo e limitação da flexão do joelho e articulação coxo-femural desse membro. Com interrogatórios concluiu-se que o desencadeamento da crise foi devido a trauma repetitivo na coxa esquerda: o paciente todos os dias brincava com seus amigos da escola de "Paulistinha", que é uma brincadeira de dois indivíduos frente a frente, o mais rápido tenta espancar a coxa do adversário com seu joelho. Devido a essa crise, o etidronato dissódico foi ajustado para $800 \mathrm{mg} / \mathrm{dia}$ e, depois, devido à pequena resposta, foi iniciada terapêutica endovenosa do bifosfonado clodronato dissódico, $300 \mathrm{mg} / \mathrm{dia}$, por 5 dias, observando-se uma boa resposta nessas primeiras 5 aplicações. O paciente melhorou a postura de deambulação, desapareceu a dor e aos poucos o edema. A família relata sensível melhora no relacionamento familiar e no rendimento escolar. O paciente receberá 5 injeções de clodronato dissódico, de $300 \mathrm{mg}$ por mês durante 5 meses, na expectativa de obter ainda melhor qualidade de deambulação.

\section{Caso 2}

R.H.N.B., 15 anos e 6 meses, feminino, nascido a termo sem intercorrências, pai portador de MOP, assintomática até 5 anos de idade, mas com estigmas clínicos da doença: implantação baixa do polegar direito, clinodactilia, osteófito lateral no polegar esquerdo, halux valgo bilateral. Aos 6 anos apresentou queixas de dor e limitação de movimentos do pescoço, acordando à noite com dores nas regiões afetadas, quando foi constatada, através de radiografia, ossificação supraclavicular. Iniciado tratamento com ácido ascórbico, a mãe referiu a melhora do psiquismo, normalização do sono e paralisação dos processos inflamatórios.

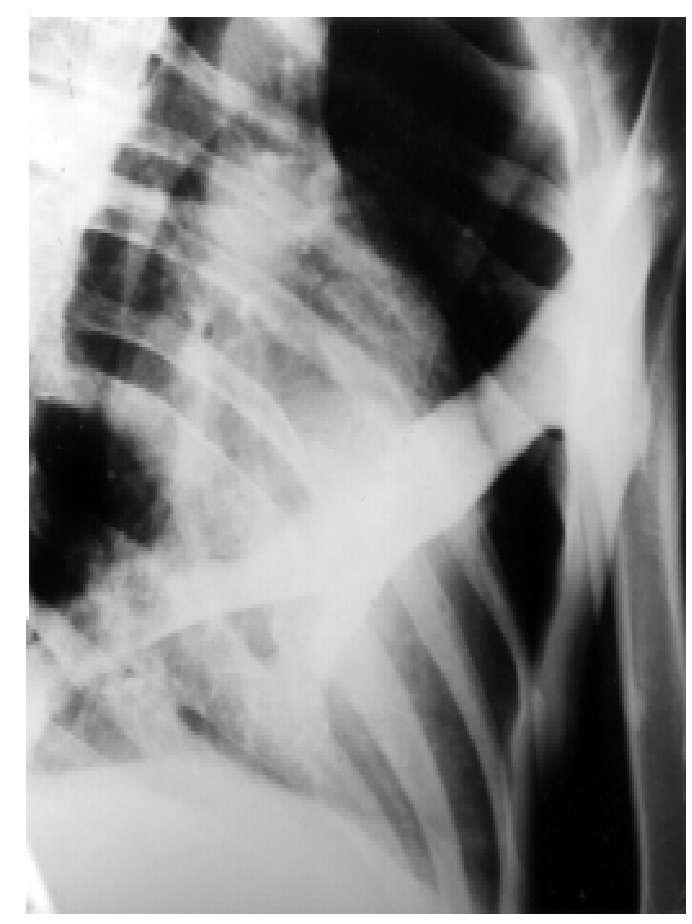

Figura 1 - Radiografia de tórax em oblíqua esquerda; extensa ossificação das estruturas de tecidos moles da parede torácica 
Tratamento com ácido ascórbico $500 \mathrm{mg} / 4$ doses ao dia continuadamente e $20 \mathrm{mg} / \mathrm{kg} / \mathrm{dia}$ de etidronato dissódico, por 2 semanas, seguido por 10 semanas na dose de $10 \mathrm{mg} /$ $\mathrm{kg} / \mathrm{dia}$, com intervalo entre os ciclos de 12 semanas, prescrito recentemente ${ }^{11}$.

\section{Caso 3}

R.D., 17 anos (1977). Natural de Belarus, União Soviética. Tratamento orientado por correspondência. Até 14 anos de idade era assintomático, quando apresentou tumoração óssea mandibular esquerda, sendo realizada cirurgia plástica. Após esse período, houve progresso da doença, surgindo outras ossificações, nos ombros e membros. Além dos achados do exame físico, apresentava-se apático, sono inquieto e com dores nas regiões comprometidas pelas ossificações. Paciente com assimetria facial proeminente, hipertrofia à esquerda e lóbulos das orelhas hipoplásicos. Polegares curtos e largos. Luxação das cabeças do fêmures e exostose no fêmur direito. Pés pequenos e largos com os primeiros metatarsos curtos. Três anos após a manifestação da doença (setembro/1997), iniciou o tratamento contínuo com ácido ascórbico, $500 \mathrm{mg} / 4$ doses ao dia. Após aproximadamente 8 meses de tratamento (junho/1998), a mãe relatou melhora significativa, com diminuição parcial das ossificações, as dores haviam desaparecido, melhora do sono e principalmente do psiquismo, com progresso no rendimento escolar, havendo ingressado no Medical College.

\section{Caso 4}

G.L.G., 6 anos de idade, masculino, nascido a termo em boas condições. Sintomatologia inicial no período neonatal, com crises de edema no pescoço, seguidas de calcificações, limitações do movimento e dificuldade de abrir a boca. Apresenta clinodactilia, sindactilia, alterações da coluna cervical e discreta limitação dos movimentos dos membros inferiores (Figura 2). Diagnóstico firmado em 1997. Fosfatase alcalina: 335 UI/L (Ref.: 90-300UI/L). Foi iniciado tratamento há 5 meses com ácido ascórbico (500 $\mathrm{mg} / 4$ vezes ao dia) e etidronato dissódico ( $200 \mathrm{mg} / \mathrm{dia}$; ciclo de 12 semanas de tratamento e 12 semanas de descanso). Em outubro de 2000 foi aumentada a dose de etidronato dissódico para $400 \mathrm{mg} /$ dia e estamos aguardando resultados de melhora por um período mínimo de um ano de tratamento.

\section{Comentários}

O diagnóstico correto e precoce da MOP é essencial para proporcionar o indício de um manejo apropriado. Embora as possibilidades terapêuticas farmacológicas sejam limitadas, a atenção ao diagnóstico pelos sinais clínicos que podem caracterizar a doença evitará biópsias e cirurgias desnecessárias, injeções intramusculares ou endovenosas, que podem piorar o prognóstico da doença, pois o trauma desencadeia ou acelera o processo inflamatório da MOP, que é uma fase precedente das calcificações ectópicas. As vacinas intramusculares, como antidifteria-tétanopertussis, sarampo, hepatite-B, etc., podem ser aplicadas subcutaneamente $^{9}$. Os tratamentos dentários devem ser cautelosos, evitando-se as anestesias, principalmente a mandibular, para prevenir ancilose da articulação têmporomandibular ${ }^{10}$. A profilaxia das cáries dentárias é essencial para evitar procedimentos mais agressivos.

Após um trauma, o paciente apresenta edema, calor moderado local, bem como aumento da VHS e fosfatase alcalina sérica (caso 4). A MOP é vista na radiografia simples aproximadamente 2 a 4 semanas após o início do processo. A calcificação inicia-se na periferia e progride em direção ao centro, o que a diferencia do osteossarcoma. A tomografia computadorizada ajuda a delinear na MOP a radioluscência central circundada pela densidade periféri$\mathrm{ca}^{13}$.

Quanto aos tratamentos até agora empregados, o uso dos bifosfonados tem eficácia nas ossificações já estabelecidas $^{11}$.

O uso de ácido ascórbico na MOP parece agir na estabilização da doença ${ }^{8}$. Conforme relato dos familiares dos pacientes que fizeram parte deste estudo, o controle da sintomatologia dolorosa tem efeito não somente no maior conforto, como na melhor qualidade de vida e de relacionamentos, sono repousante e maior rendimento escolar.

A diminuição das ossificações, possivelmente em transição, isto é, ainda em fase de processo inflamatório, como observado nos casos 1 e 3, tem sido atribuída à ação do ácido ascórbico na modulação da síntese do procolágeno III $^{8}$.

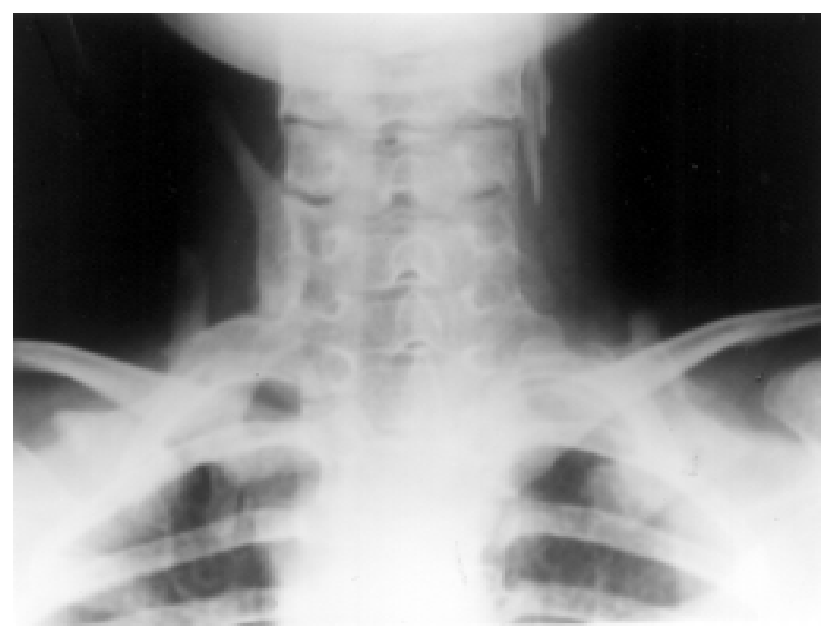

Figura 2 - Radiografia em AP cérvico-torácica evidenciando a distribuição inicial da ossificação dos tecidos moles: cervical, ombros (interescapular) e tórax 
Nos casos de MOP grave com limitação dos movimentos e intolerância gástrica (caso 1), o uso de difosfonado endovenoso pode estar indicado e ter boa melhora, como também observado por Alpigiani et al. ${ }^{12}$, em 3 pacientes que recuperaram a capacidade motora utilizando-se $300 \mathrm{mg} /$ dia de ácido diclorometilfosfônico, 10 doses ao mês por 5 meses.

O trauma repetitivo é o maior fator desencadeante da doença, mesmo sob uso do ácido ascórbico em altas doses, como observado no paciente 1 . A atividade física mais adequada aos pacientes com MOP é a natação, mesmo com algumas limitações, pois o paciente pode se adequar com os movimentos que lhe sobram, tendo assim uma forma de realizar exercícios físicos sem as lesões oriundas de impactos ou trauma direto.

O diagnóstico diferencial se faz com a calcinose idiopática universal, dermatomiosite, calcinose idiopática tumoral e doenças por alterações no metabolismo do cálcio. A presença de ossificações nos tecidos moles do esqueleto axial e anomalias nos dedos simplifica o diagnóstico nos casos de MOP.

Os pacientes com MOP, quando jovens, devem ser fiscalizados e orientados para evitarem traumas. Nossa opinião é que a associação de ácido ascórbico e bifosfonado por via oral ou endovenosa pode ser uma alternativa no controle e diminuição das calcificações ectópicas a longo prazo, melhorando significativamente a sua qualidade de vida. Nos pacientes com quadro clínico grave e limitação da deambulação, o uso de bifosfonado endovenoso foi uma alternativa de melhora mais rápida do quadro clínico.

\section{Referências bibliográficas}

1. Whyte MP. Heritable metabolic and dysplastic bone diseases. Endocrinol Metabol Clin N Am 1990; 19: 163-73.

2. Patin G. Lettres choisis de feu M. Guy Patin. Letter of August 27, 1648, to AF. Cologne P. du Laurens, 1692. Tome 1, vol. 5, p. 28.
3. Reinig JW, Hill SC, Fang M, Marini J, Zasloff MA. Fibrodysplasia Ossificans Progressiva: CT Appearance. Radiology 1986;159: 153-7.

4. Smith R, Russel RGG, Woods CG. Myositis Ossificans Progressiva. Clinical features of eight patients and their response to treatment. J Bone Joint Surg 1976; 58: 48-57.

5. Rogers JG, Dorst JP, Geho BW. Use and complications of highdose disodium etidronate therapy in fibrodysplasia ossificans progressiva. J Pediatr 1977; 91: 1011-14.

6. Bar Oz B, Boneh A. Myositis ossificans progressiva: a 10 year follow-up on a patient treated with etidronate disodium. Acta Paediatr 1994; 83: 1332-34.

7. McEvoy GK. Unclassified therapeutic agents. In:__. American Hospital Formulary Service (AHFS) Drug Information. Bethesda, Library of Congress, 2000, 3399-403.

8. Palhares DB. Myositis Ossificans Progressive. Calcif Tissue Int 1997; 60: 394.

9. Buyse G, Silberstein J, Goemans N, Casaer P. Fibrodysplasia Ossificans Progressiva: still turning into wood after 300 years? Eur J Pediatr 1995; 154: 694-99.

10. Connor JM, Evans DAP. Fibrodysplasia Ossificans Progressiva. The clinical features and natural history of 34 patients. J Bone Joint Surg 1982; 64-B: 76-83.

11. Bar B, Boneh A. Myositis ossificans progressiva: a 10-year follow-up on a patient treated with etidronate disodium. Acta Paediatr 1994; 83: 1332-34.

12. Alpigiani MG, Puleo MG, Callegarini I, Di Bella E, Debbia C, Buzzanca $\mathrm{C}$ et al. $\mathrm{L}$, acido diclorometilenbifosfonico nella terapia della miosite ossificante progressiva. Minerva Pediatr 1996; 48: $159-63$.

13. Bullough P. Orthopaedic Pathology. $3^{\mathrm{a}}$ ed. London: Times Mirror International Publishers Limited; 1997.

Endereço para correspondência:

Dr. Durval Palhares

Rua Maria Madalena, 319 - Bairro Rosa Pires

CEP 79004-360 - Campo Grande, MS

Fax: (67) 7879232

E-mail:dbatista@nin.ufms.br 